\title{
Deformation of a Uniform Half-Space with Rigid Boundary Due to a Strike-Slip Line Source
}

\author{
Meenal Malik ${ }^{1}$ and Mahabir Singh ${ }^{2}$ \\ ${ }^{1}$ Department of Applied Sciences, Matu Ram Institute of Engineering and Management, Rohtak-12400, \\ Haryana, India \\ ${ }^{2}$ CoE, Deenbandhu Chhotu Ram University of Science and Technology, Murthal, Sonepat-131039, Haryana, \\ India
}

\begin{abstract}
Closed-form analytical expressions for the displacement and stresses at an arbitrary point caused by strike-slip line source buried in a homogeneous, isotropic, perfectly elastic half-space with rigid boundary are obtained. These expressions are used, further, to find the expressions for the displacement and stresses caused by vertical as well as horizontal strike-slip line source. The variation of the displacement and stress fields due to vertical strike-slip line source and horizontal strike-slip line source with distance from the fault and depth from the fault is studied numerically.

Keywords - Half-space, rigid boundary, static deformation, strike-slip faulting
\end{abstract}

\section{Introduction}

The theory of dislocation was first introduced to the field of seismology by Steketee (1958a, b). A dislocation is considered to be a defect in an elastic (or viscoelastic) medium which is represented by a discontinuity in the displacement field. Static dislocation models are used to analyze the static deformation of the medium caused by earthquake faults. These faults are slip planes across which discontinuous motion occurs in the Earth. If the slip on the fault is parallel to the fault strike, the fault is known as strike-slip fault. Steketee (1958a, b) obtained the displacement and stress field of a strike-slip fault with uniform slip. He gave the method for construction of the Green's functions and calculated one set of Green's functions corresponding to a vertical strike-slip fault. Following Steketee, Chinnery (1961) derived closed- form analytic expressions for the displacement field in the vicinity of a vertical, rectangular, strike-slip fault and obtained contour maps for two representative cases: Tango and North Idu earthquakes and the San Andreas Fault. Press (1965) computed residual displacements, strains and tilts at intermediate and large distances from major earthquakes due to vertical, rectangular, strike-slip and dip-slip faults in a half-space. The static deformation of various Earth models caused by two-dimensional sources has been studied by many investigators. Maruyama (1966) obtained the displacement and stress fields corresponding to long strike-slip and dip-slip faults of arbitrary dip. Rybicki (1971) investigated the effect of a vertical and a horizontal discontinuity in a half-space on the elastic residual field of a long strike-slip fault using the method of images. Sato (1971) and Sato and Yamashita (1975) derived the expressions for the static surface deformations due to two-dimensional strike slip and dip-slip faults located along the dipping boundary between the two different media. McHugh and Johnston (1977) and Rybicki and Kasahara (1977) studied the static deformation of a laterally inhomogeneous medium by a two- dimensional strike-slip fault. Rybicki (1978) derived the formulae for the displacement, strain and stress fields of a twodimensional strike-slip fault in a laterally inhomogeneous half-space. Mahrer and Nur (1979a) developed a model for a very long strike-slip fault in a non- homogeneous half-space with a downward varying shear modulus. Mahrer and Nur (1979b) considered a static two-dimensional model of a long strike-slip fault in a crust with horizontally varying rigidity and calculated the surface displacement and strain fields for a number of slip regimes and crustal rigidity profiles. Wang and Wu (1983), following Steketee's method of integration, obtained a closed-form analytical solution for the displacement and stress field due to a "trapezodial" type of non-uniform slip along a strike-slip fault in an elastic half-space.

Singh (1985) considered the problem of the static deformation of a multilayered half-space by a long strike-slip dislocation. The representation of sources corresponding to antiplane strain has been considered. Singh and Garg (1986) obtained the integral expressions for the Airy stress function in an unbounded medium due to various two-dimensional seismic sources. Sharma and Garg (1991) have obtained the deformation field at any point of a layered half-space due to very long strike-slip dislocations. Madan and Garg (1997) obtained the analytic expressions for the displacement and stresses at any point of an orthotropic horizontal elastic layer coupling in different ways to a base due to a very long inclined strike-slip fault situated in a layer. Kumar et al. (2002) studied static deformation of two monoclinic elastic half-spaces in welded contact due to a long inclined strike-slip fault situated in one of the half-spaces analytically and numerically. Chug et al. (2010) obtained closed-form analytical expressions for the deformation at any point of a two-phase medium consisting of a 
homogeneous orthotropic half-space in welded contact with a homogeneous isotropic elastic half-space caused by non-uniform slip along a very long vertical strike-slip dislocation, situated in the orthotropic elastic halfspace. Singh et al. (2011) obtained analytical expressions for stresses at an arbitrary point of homogeneous, isotropic, perfectly elastic half-space with rigid boundary caused by a long tensile fault of finite width. Malik et al. (2012) obtained analytical expressions for stresses and the displacements at an arbitrary point of homogeneous, isotropic, perfectly elastic half-space with rigid boundary caused by a long dip-slip fault.

Beginning with the expression for the displacement in an infinite medium given by Singh (1985), we have obtained the integral expressions for the displacement and stresses due to a long strike-slip fault in a homogeneous, isotropic, perfectly elastic half-space by applying the boundary conditions of rigid boundary at the surface of the half-space. The integrals were then evaluated analytically, obtaining closed-form expressions for the displacement and the stresses at any point of the half-space caused by a long strike-slip fault. The expressions for the displacement and stresses for a vertical and a horizontal strike-slip dislocation follow immediately.

\section{Theory}

In the Cartesian co-ordinates system $(x, y, z) \equiv\left(x_{1}, x_{2}, x_{3}\right)$, the equations of equilibrium are

$p_{i j, j}+f_{i}=0 \quad(\mathrm{i}=1,2,3)$

where $p_{i j}$ is the stress tensor, $f_{i}$ is the body force per unit volume and

$p_{i j, j} \equiv \frac{\partial}{\partial x_{j}} p_{i j}$

Summation over the repeated suffix is understood. The stress-strain relations for an isotropic medium are

$p_{i j}=\lambda \delta_{i j} e_{k k}+2 \mu e_{i j}$

where $\delta_{i j}$ is the Kronecker delta $\left(\delta_{i j}=1\right.$ for $i=j$ and $\delta_{i j}=0 i \neq j$ ), $\lambda$ and $\mu$ are the lame constants and $e_{i j}$ is the strain tensor. If $\left(u_{1}, u_{2}, u_{3}\right)$ denote the components of the displacement vector, the strain-displacement relations are

$e_{i j}=\frac{1}{2}\left(u_{i, j}+u_{j, i}\right)$

Using equations (1.2) and (1.3), we obtain the stress-displacement relations

$p_{i j}=\lambda \delta_{i j} u_{k, k}+\mu\left(u_{i, j}+u_{j, i}\right)$

We shall be considering a two-dimensional approximation in which the displacement components and consequently, the stresses are independent of a single co-ordinate, say $x_{1}$, so that $\partial / \partial x_{1} \equiv 0$. Under this assumption. equations (1.1) - (1.4) get decoupled into two mutually independent sets- one representing the antiplane strain case $\left(u_{2}=u_{3}=0\right)$ and the other the plane strain case $\left(u_{1}=0\right)$.

For antiplane case, we have

\section{Antiplane Strain}

$u_{1}=u_{1}(y, z), \quad u_{2}=u_{3}=0$

The non-zero strain and stress components are

$e_{12}=\frac{1}{2} \frac{\partial u_{1}}{\partial y}, \quad e_{13}=\frac{1}{2} \frac{\partial u_{1}}{\partial z}$

$p_{12}=\mu \frac{\partial u_{1}}{\partial y}, \quad p_{13}=\mu \frac{\partial u_{1}}{\partial z}$

For zero body forces, equilibrium equations (1.1b) and (1.1c) are identically satisfied and (1.1a) gives, using equation (1.6),

$\nabla^{2} u_{1}=0$

where $\nabla^{2}$ is the two-dimensional Laplacian differential operator.

A solution of equation (1.7) corresponding a line source at $(0,0, \mathrm{~h})$ in an infinite medium is (Singh, 1985): 
$u_{1}^{(0)}=\int_{0}^{\infty}\left[A_{0} \sin k y+B_{0} \cos k y\right] e^{-k|z-h|} d k$

where the source coefficients $A_{0}$ and $B_{0}$ are independent of k. Singh (1985) has obtained these coefficients for the single couple (12) and (13). For the single couple (12)

$A_{0}=\frac{F_{12}}{2 \pi \mu}, \quad B_{0}=0$

while for the single couple (13)

$A_{0}=0, \quad B_{0}= \pm \frac{F_{13}}{2 \pi \mu}$

where the upper sign is for $\mathrm{z}>\mathrm{h}$ and the lower $\operatorname{sign}$ for $\mathrm{z}<\mathrm{h}$.

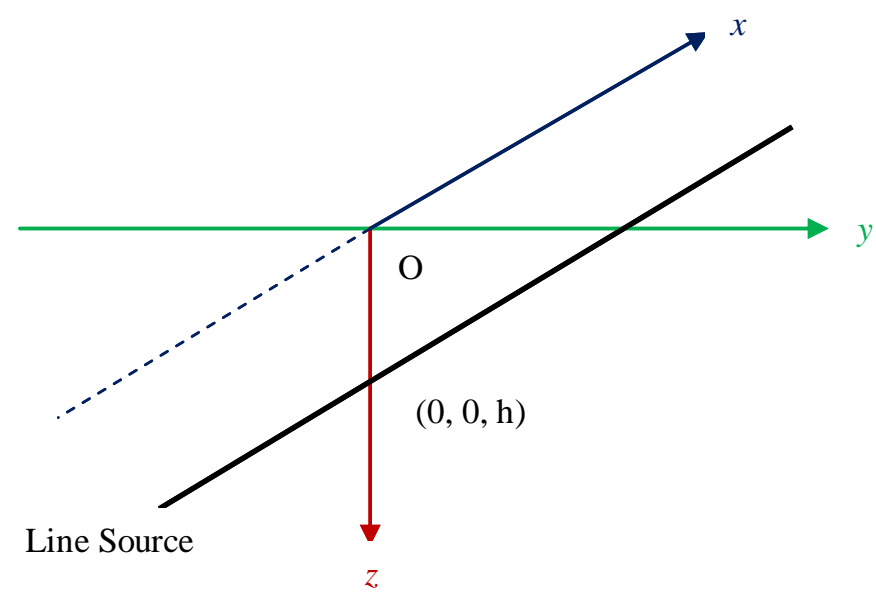

Fig.1 A uniform half-space $(z \geq 0)$ with a line source acting at the point $(0,0, \mathrm{~h})$

For a line source parallel to the $x$-axis acting at the point $(0,0, \mathrm{~h})$ of the half space $z \geq 0$ (Figure 1), a suitable solution of equation (1.7) is of the form

$u_{1}=u_{1}^{(0)}+\int_{0}^{\infty}[A \sin k y+B \cos k y] e^{-k z} d k$

where $u_{1}^{(0)}$ is given by the equation (1.8) and $\mathrm{A}$ and $\mathrm{B}$ are unknown functions of $\mathrm{k}$ to be determined from the boundary conditions. From equations (1.6) and (1.10), the stresses are found to be

$$
\begin{aligned}
& p_{12}=\mu \int_{0}^{\infty}\left(A_{0} e^{-k|z-h|}+A e^{-k z}\right) \cos k y k d k-\mu \int_{0}^{\infty}\left(B_{0} e^{-k|z-h|}+B e^{-k z}\right) \sin k y k d k \\
& p_{13}=\mu \int_{0}^{\infty}\left(\mp A_{0} e^{-k|z-h|}-A e^{-k z}\right) \sin k y k d k+\mu \int_{0}^{\infty}\left(\mp B_{0} e^{-k|z-h|}-B e^{-k z}\right) \cos k y k d k
\end{aligned}
$$

where the upper sign is for $\mathrm{z}>\mathrm{h}$ and the lower $\operatorname{sign}$ for $\mathrm{z}<\mathrm{h}$.

We assume that the surface of the half-space $z \geq 0$ is with rigid boundary. Therefore, the boundary condition is $u_{1}=0$ at $\mathrm{z}=0$

It is noticed from equations $(1.9 \mathrm{a}, \mathrm{b})$ that $A_{0}$ and $B_{0}$ have different values for $\mathrm{z} \stackrel{>}{<}$ h. Let $A^{-}$and $B^{-}$be respectively, the values of $A_{0}$ and $B_{0}$ for $\mathrm{z}<$ h. From equation (1.10) and (1.13), we get

$$
\begin{aligned}
& A=-A^{-} e^{-k h} \\
& B=-B^{-} e^{-k h}
\end{aligned}
$$

Putting the values of A and B in equations (1.10) - (1.12), we get the following expressions for the displacement and stresses at any point of the half-space. 


$$
\begin{aligned}
& u_{1}=\int_{0}^{\infty}\left[A_{0} \sin k y+B_{0} \cos k y\right] e^{-k|z-h|} d k-\int_{0}^{\infty}\left[A^{-} \sin k y+B^{-} \cos k y\right] e^{-k(z+h)} d k \\
& p_{12}=\mu \int_{0}^{\infty}\left[A_{0} \cos k y-B_{0} \sin k y\right] e^{-k|z-h|} k d k-\mu \int_{0}^{\infty}\left[A^{-} \cos k y-B^{-} \sin k y\right] e^{-k(z+h)} k d k \\
& p_{13}=\mu \int_{0}^{\infty}\left(A_{0} \sin k y+B_{0} \cos k y\right) e^{-k|z-h|} k d k+\mu \int_{0}^{\infty}\left(A^{-} \sin k y+B^{-} \cos k y\right) e^{-k(z+h)} k d k
\end{aligned}
$$

Evaluating the integrals by using standard integral transform tables (Appendix I), we obtain

$$
\begin{aligned}
& u_{1}=\frac{A_{0} y}{R^{2}}+\frac{B_{0}(h-z)}{R^{2}}-\frac{A^{-} y}{S^{2}}-\frac{B^{-}(h+z)}{S^{2}} \\
& p_{12}=\mu\left[\frac{A_{0}}{R^{2}}\left\{\frac{2(h-z)^{2}}{R^{2}}-1\right\}-\frac{2 B_{0} y(h-z)}{R^{4}}-\frac{A^{-}}{S^{2}}\left\{\frac{2(h+z)^{2}}{S^{2}}-1\right\}+\frac{2 B^{-} y(h+z)}{S^{4}}\right] \\
& p_{13}=\mu\left[\frac{2 A_{0} y(h-z)}{R^{4}}+\frac{B_{0}}{R^{2}}\left\{\frac{2(h-z)^{2}}{R^{2}}-1\right\}+\frac{2 A^{-} y(h+z)}{S^{4}}+\frac{B^{-}}{S^{2}}\left\{\frac{2(h+z)^{2}}{S^{2}}-1\right\}\right]
\end{aligned}
$$

\section{Strike-Slip Dislocation}

Let $u_{i}^{j}$ denote the $x_{j}$-component of the displacement at $P\left(x_{2}, x_{3}\right)$ caused by a unit concentrated force acting at $Q\left(y_{2}, y_{3}\right)$ in the $x_{i}$ - direction. Then, for antiplane strain, the displacement field due to a long displacement dislocation of arbitrary orientation is given by (Maruyama, 1966)

$u_{1}=\int_{L} \Delta u_{1} u_{1 k}^{1} n_{k} d s$

where $u_{1 k}^{1}=\mu \frac{\partial}{\partial y_{k}} u_{1}^{1}$

From equation (1.22), we note that $u_{1 k}^{1}$ is the displacement at $P\left(x_{2}, x_{3}\right)$ in the $x_{1}$-direction caused by a source, specified by $[1 \mathrm{k}]$, we observe that

$$
\left.\begin{array}{l}
{[12]=\mu(12)} \\
{[13]=\mu(13)}
\end{array}\right\}
$$

Equation (1.23) shows that the sources [12] and [13] represent single couples with arms in the $x_{2}$ - and $x_{3}$ directions, respectively.

Writing $\Delta u_{1}=b, n_{2}=-\sin \delta, \quad n_{3}=\cos \delta$, where $\delta$ is the dip of the fault, equation (1.21) becomes

$$
u_{1}=\int_{L} b\left(u_{13}^{1} \cos \delta-u_{12}^{1} \sin \delta\right) d s
$$

Thus the source [12] corresponds to a vertical strike-slip fault $\left(\delta=90^{\circ}\right)$ and the source [13] corresponds to a horizontal strike-slip fault ( $\delta=0^{0}$ ). From equations (1.23) and (1.24), we have

$u_{1}=\int_{L} \mu b\left(u_{(13)}^{1} \cos \delta-u_{(12)}^{1} \sin \delta\right) d s$

In case of a line dislocation, we can do away with the integration in equation (1.25a), thus obtaining $u_{1}=\mu b d s\left(u_{(13)}^{1} \cos \delta-u_{(12)}^{1} \sin \delta\right)$

Therefore, the field due to a long strike-slip fault of arbitrary dip can be expressed in terms of the fields due to a vertical strike-slip fault and a horizontal strike- slip fault.

\section{Vertical Strike-Slip}


From equation (1.25b), the single couple (12) of moment $F_{12}$ is equivalent to a vertical strike-slip line source such that

$F_{12}=\mu b d s$

where $b$ is the slip. Therefore, from equation (1.9a), the source coefficients for a vertical strike-slip source are given by

$A_{0}=A^{-}=\frac{b d s}{2 \pi}, \quad B_{0}=B^{-}=0$

On putting these values of the source coefficients from equation (1.27) into equations (1.18-1.20), the results for the displacement and the stresses for a vertical strike-slip are found to be

$u_{1}=\frac{b d s y}{2 \pi}\left[\frac{1}{R^{2}}-\frac{1}{S^{2}}\right]$

$p_{12}=\frac{\mu b d s}{2 \pi}\left[\frac{2(h-z)^{2}}{R^{4}}-\frac{1}{R^{2}}-\frac{2(h+z)^{2}}{S^{4}}+\frac{1}{S^{2}}\right]$

$p_{13}=\frac{\mu b d s y}{\pi}\left[\frac{(h-z)}{R^{4}}+\frac{(h+z)}{S^{4}}\right]$

\section{Horizontal Strike-Slip}

The single couple (13) of moment $F_{13}$ is equivalent to a horizontal strike-slip line source such that $F_{13}=\mu b d s$

Therefore, from equation (1.9b), the source coefficients for a horizontal strike-slip source are given by

$$
A_{0}=A^{-}=0, \quad B_{0}= \pm \frac{b d s}{2 \pi}, \quad B^{-}=-\frac{b d s}{2 \pi}
$$

On putting these values of the source coefficients from equation (1.32) into equations (1.18-1.20), the results for the displacement and the stresses for a horizontal strike-slip are found to be

$$
\begin{aligned}
& u_{1}=\frac{b d s}{2 \pi}\left[\frac{(z-h)}{R^{2}}+\frac{(z+h)}{S^{2}}\right] \\
& p_{12}=\frac{\mu b d s y}{\pi}\left[\frac{(h-z)}{R^{4}}-\frac{(h+z)}{S^{4}}\right] \\
& p_{13}=\frac{\mu b d s}{2 \pi}\left[\frac{1}{R^{2}}-\frac{2(h-z)^{2}}{R^{4}}+\frac{1}{S^{2}}-\frac{2(h+z)^{2}}{S^{4}}\right]
\end{aligned}
$$

where $R^{2}=y^{2}+(z-h)^{2}, \quad S^{2}=y^{2}+(z+h)^{2}$.

\section{Numerical Results}

We study numerically displacement and the stress fields at any point of the uniform isotropic perfectly elastic half-space with rigid boundary caused by a vertical and horizontal strike-slip line source. We define the following dimensionless quantities

$$
Y=\frac{y}{L}, \quad Z=\frac{z}{L}
$$

where $\mathrm{L}$ is the distance of the line source from the interface. Let the dimensionless displacement and stresses be denoted by $U_{i}$ and $P_{i j}$. Then,

$U_{i}=\frac{\pi L}{b d s} u_{i}, \quad P_{i j}=\frac{\pi L^{2}}{\mu b d s} p_{i j}$

where $b$ is the magnitude of dislocation (slip) and ds is the width of the fault. 
From equations (1.28-1.30), (1.36) and (1.37), we get the following expressions for the dimensionless displacement and stresses for a vertical strike-slip line source:

$$
\begin{aligned}
& U_{1}=\frac{Y}{2}\left[\frac{1}{A^{2}}-\frac{1}{B^{2}}\right] \\
& P_{12}=\frac{1}{2}\left[\frac{2(1-Z)^{2}}{A^{4}}-\frac{1}{A^{2}}-\frac{2(1+Z)^{2}}{B^{4}}+\frac{1}{B^{2}}\right] \\
& P_{13}=Y\left[\frac{(1-Z)}{A^{4}}+\frac{(1+Z)}{B^{4}}\right]
\end{aligned}
$$

From equations (1.33-1.35), (1.36) and (1.37), we get the following expressions for the dimensionless displacement and stresses for a horizontal strike-slip line source:

$$
\begin{aligned}
& U_{1}=\frac{1}{2}\left[\frac{(Z-1)}{A^{2}}+\frac{(Z+1)}{B^{2}}\right] \\
& P_{12}=Y\left[\frac{(1-Z)}{A^{4}}-\frac{(1+Z)}{B^{4}}\right] \\
& P_{13}=\frac{1}{2}\left[\frac{1}{A^{2}}-\frac{2(1-Z)^{2}}{A^{4}}+\frac{1}{B^{2}}-\frac{2(1+Z)^{2}}{B^{4}}\right]
\end{aligned}
$$

where $A^{2}=Y^{2}+(Z-1)^{2}, \quad B^{2}=Y^{2}+(Z+1)^{2}$

\section{Discussion}

Figures 2(a) - 2(c) show the variation of dimensionless vertical displacement $U_{1}$ and shear stresses $P_{12}$ and $P_{13}$ at the interface with the horizontal distance from the fault. Figure 2(a) shows the variation of displacement $U_{1}$ with distance from the fault at $\mathrm{z}=2 \mathrm{~L}, 2.5 \mathrm{~L}$ and $3 \mathrm{~L}$ respectively. Moreover, $U_{1}$ tends to zero as y approaches to infinity. Figure 2(b) shows the variation of the dimensionless shear stress $P_{12}$ with the horizontal distance from the fault at $\mathrm{z}=2 \mathrm{~L}, 2.5 \mathrm{~L}$ and $3 \mathrm{~L}$ respectively. At $\mathrm{y}=0, P_{12}$ attains its maximum value for $\mathrm{z}=2 \mathrm{~L}$ and minimum value at $\mathrm{z}=3 \mathrm{~L} . \quad P_{12}$ approaches to zero as y approaches to infinity. Figure 2(c) shows the variation of the dimensionless shear stress $P_{13}$ with y at z $=2 \mathrm{~L}, 2.5 \mathrm{~L}$ and $3 \mathrm{~L}$. It is observed that $P_{13}$ is zero at $\mathrm{y}=0$ and also tends to zero as $\mathrm{y}$ approaches to infinity.

Figures 3(a) - 3(c) show the variation of dimensionless vertical displacement $U_{1}$ and shear stresses $P_{12}$ and $P_{13}$ at the interface with the depth at locations at $\mathrm{y}=2 \mathrm{~L}, 2.5 \mathrm{~L}$ and $3 \mathrm{~L}$ respectively. It is observed that for $\mathrm{y}=3 \mathrm{~L}$, the variation of $U_{1}$ is smooth but for $\mathrm{y}=2 \mathrm{~L}, U_{1}$ varies strongly in the range $0<\mathrm{z}<2 \mathrm{~L}$. Moreover it tends to zero as $\mathrm{z}$ approaches to infinity. Figure 3(b) shows the variation of the dimensionless shear stress $P_{12}$ with the depth at $\mathrm{y}=2 \mathrm{~L}, 2.5 \mathrm{~L}$ and $3 \mathrm{~L}$ respectively. The variation of $P_{12}$ for $\mathrm{y}=2 \mathrm{~L}$ depends strongly on $\mathrm{z}$ whereas for $\mathrm{y}=2.5 \mathrm{~L}$ and $\mathrm{y}=3 \mathrm{~L}$, the variation of stress component $P_{12}$ is smooth. $P_{12}$ tends to zero as $\mathrm{z}$ approaches to infinity. Figure 3(c) shows the variation of the dimensionless shear stress $P_{13}$ with $\mathrm{z}$ at $\mathrm{y}=2 \mathrm{~L}$, $2.5 \mathrm{~L}$ and $3 \mathrm{~L}$. For $\mathrm{y}=2 \mathrm{~L}, P_{13}$ attains the maximum value. The variation is significant in the range $\mathrm{o}<\mathrm{z}<3 \mathrm{~L}$. $P_{13}$ tends to zero as z approaches to infinity.

Figures 4(a) - 4(c) show the variation of dimensionless horizontal displacement $U_{1}$ and shear stresses $P_{12}$ and $P_{13}$ at the interface with the horizontal distance from the fault. Figure 4(a) shows the variation of the horizontal displacement $U_{1}$ with distance from the fault at $\mathrm{z}=2 \mathrm{~L}, 2.5 \mathrm{~L}$ and $3 \mathrm{~L}$ respectively. The value of $U_{1}$ is maximum at $\mathrm{z}=2 \mathrm{~L}$ and minimum at $\mathrm{z}=3 \mathrm{~L}$ and tends to zero as y approaches to infinity. Figures 4 (b) and 4 (c) 
show the variation of the dimensionless shear stresses $P_{12}$ and $P_{13}$ respectively with the horizontal distance from the fault at $\mathrm{z}=2 \mathrm{~L}, 2.5 \mathrm{~L}$ and $3 \mathrm{~L} . P_{12}$ attains its minimum value for $\mathrm{z}=2 \mathrm{~L}$ and maximum value at $\mathrm{z}=3 \mathrm{~L}$. $P_{12}$ approaches to zero as y approaches to infinity. The stress $P_{13}$ also attains maximum value at $\mathrm{z}=2 \mathrm{~L}$ and varies strongly in the range $0<\mathrm{y}<2 \mathrm{~L}$. It also tends to zero as y approaches to infinity.

Figures 5(a) - 5(c) show the variation of dimensionless horizontal displacement $U_{1}$ and shear stresses $P_{12}$ and $P_{13}$ at the interface with the depth at locations at $\mathrm{y}=2 \mathrm{~L}, 2.5 \mathrm{~L}$ and $3 \mathrm{~L}$ respectively. The variation of $U_{1}$ is sharp for $\mathrm{z}=2 \mathrm{~L}$ while it is smooth for $\mathrm{z}=3 \mathrm{~L}$. Moreover it tends to zero as $\mathrm{z}$ approaches to infinity. Figure 5(b) shows the variation of the dimensionless shear stress $P_{12}$ with the depth at $\mathrm{y}=2 \mathrm{~L}, 2.5 \mathrm{~L}$ and $3 \mathrm{~L}$ respectively. The variation of $P_{12}$ for $\mathrm{y}=2 \mathrm{~L}$ depends strongly on $\mathrm{z}$ whereas for $\mathrm{y}=2.5 \mathrm{~L}$ and $\mathrm{y}=3 \mathrm{~L}$, the variation of stress component $P_{12}$ is smooth. $P_{12}$ tends to zero as z approaches to infinity. Figure 5(c) shows the variation of the dimensionless shear stress $P_{13}$ with z at $\mathrm{y}=2 \mathrm{~L}, 2.5 \mathrm{~L}$ and $3 \mathrm{~L}$. The variation of the shear stress is noticeable in the range $2 \mathrm{~L}<\mathrm{z}<3 \mathrm{~L}$ for all values of $\mathrm{y}$ and tends to zero as $\mathrm{z}$ approaches to infinity.

\section{VERTICAL STRIKE SLIP FAULT}

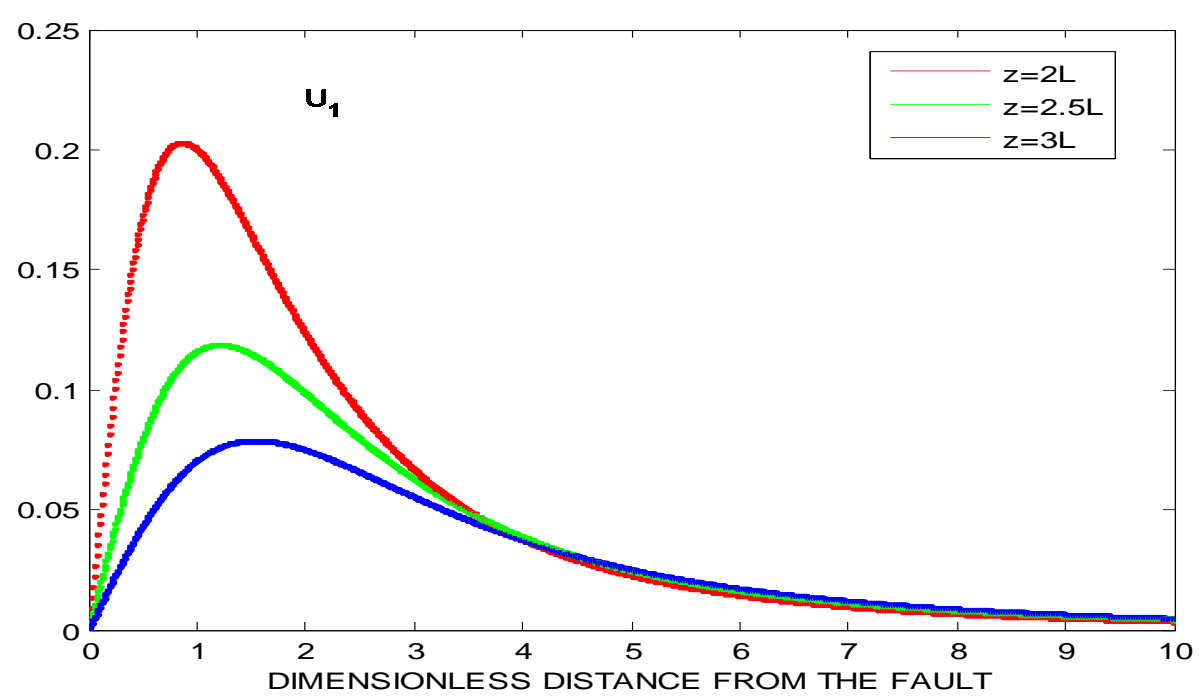

Fig. 2(a) Variation of dimensionless displacement $U_{1}$ with the distance from the fault

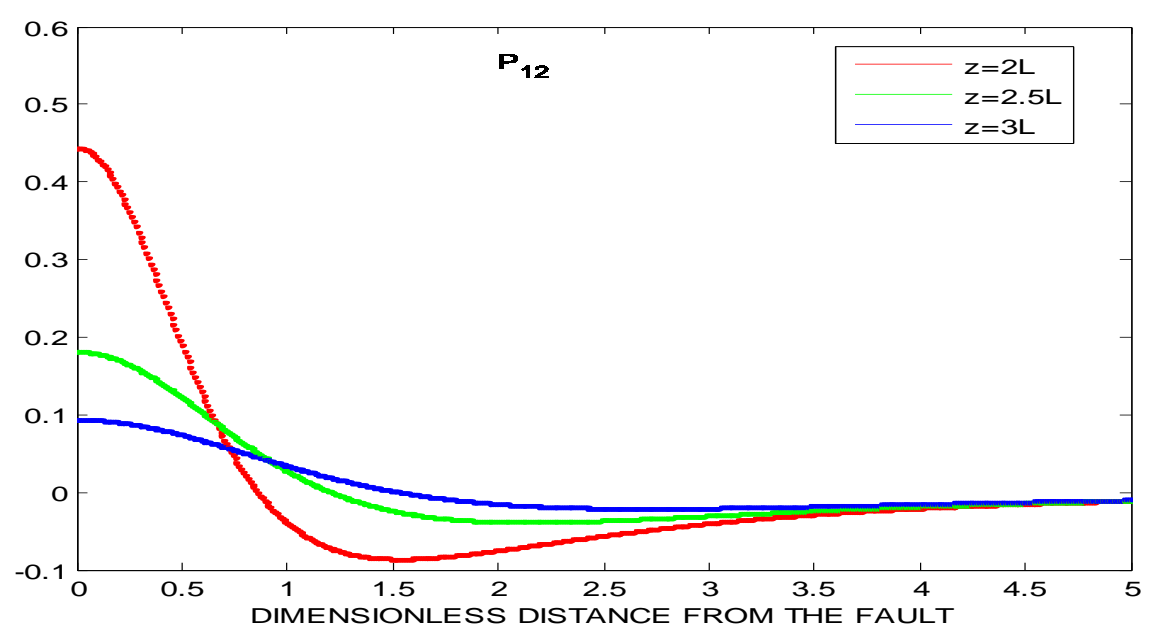

Fig. 2(b) Variation of dimensionless shear stress $\left(\mathrm{P}_{12}\right)$ with the distance from the fault 


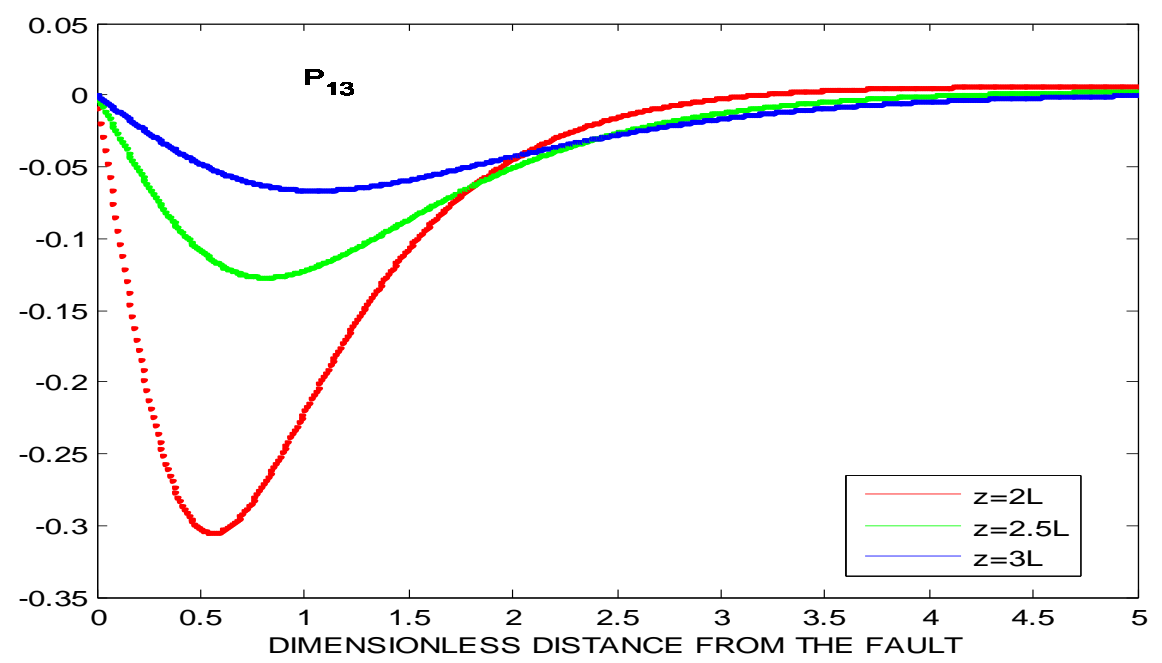

Fig. 2(c) Variation of dimensionless shear stress $\left(\mathrm{P}_{13}\right)$ with the distance from the fault

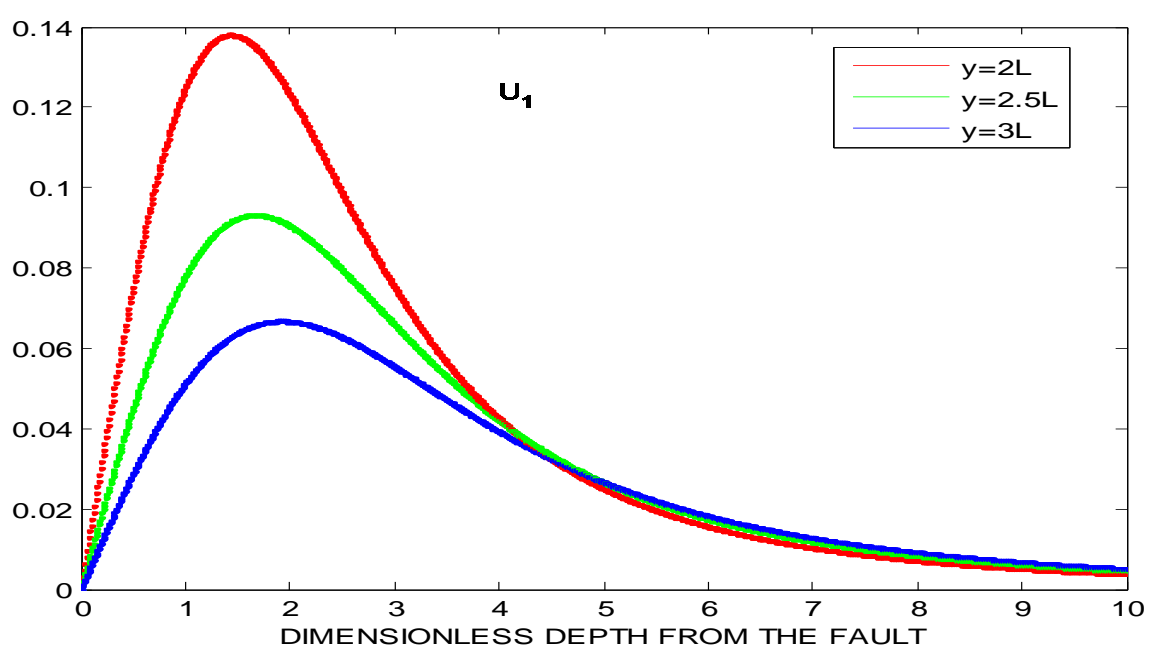

Fig. 3(a) Variation of dimensionless displacement $U_{1}$ with the depth from the fault

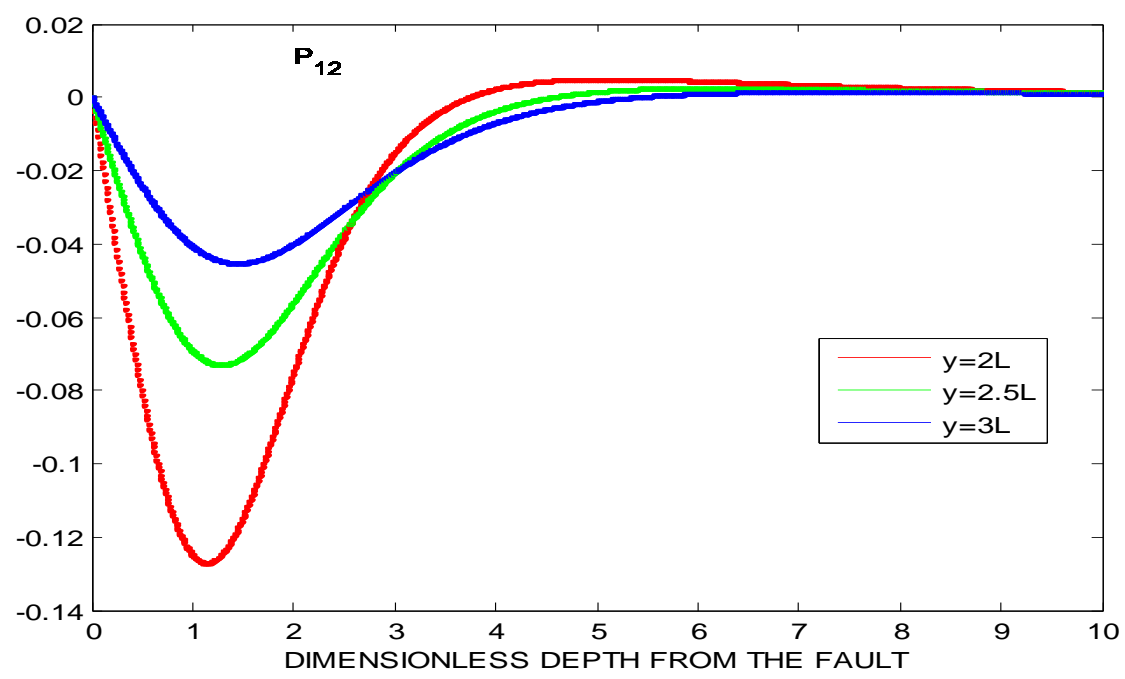

Fig. 3(b) Variation of dimensionless shear stress $\left(\mathrm{P}_{12}\right)$ with the depth from the fault 


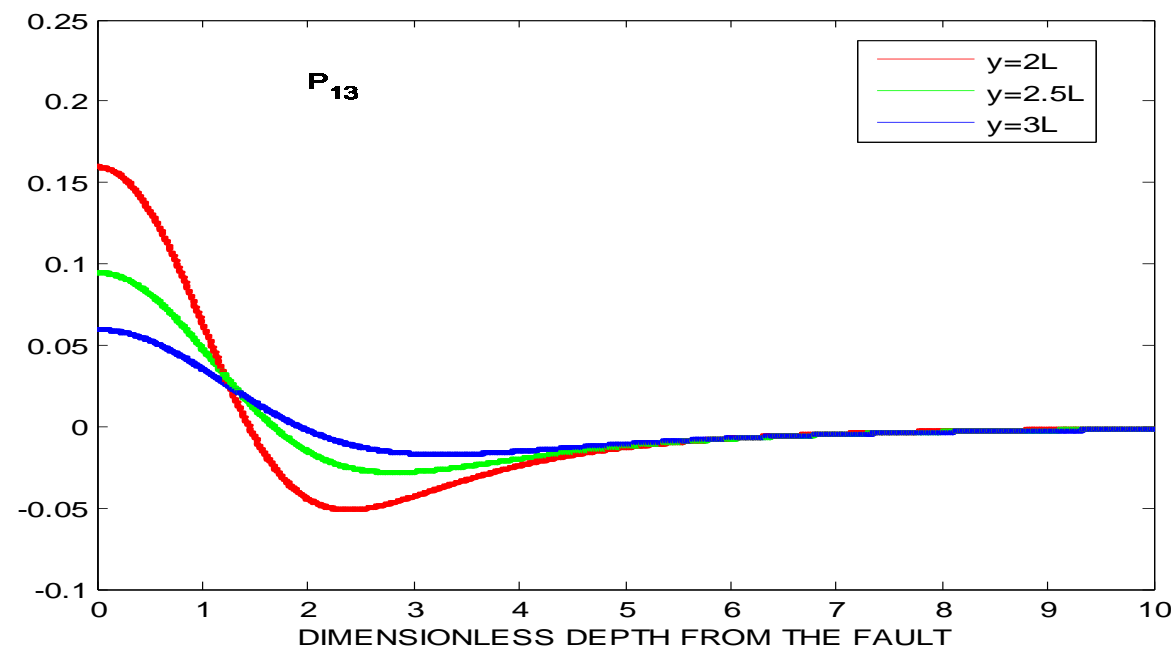

Fig. 3(c) Variation of dimensionless shear stress $\left(\mathrm{P}_{13}\right)$ with the depth from the fault

HORIZONTAL STRIKE-SLIP FAULT

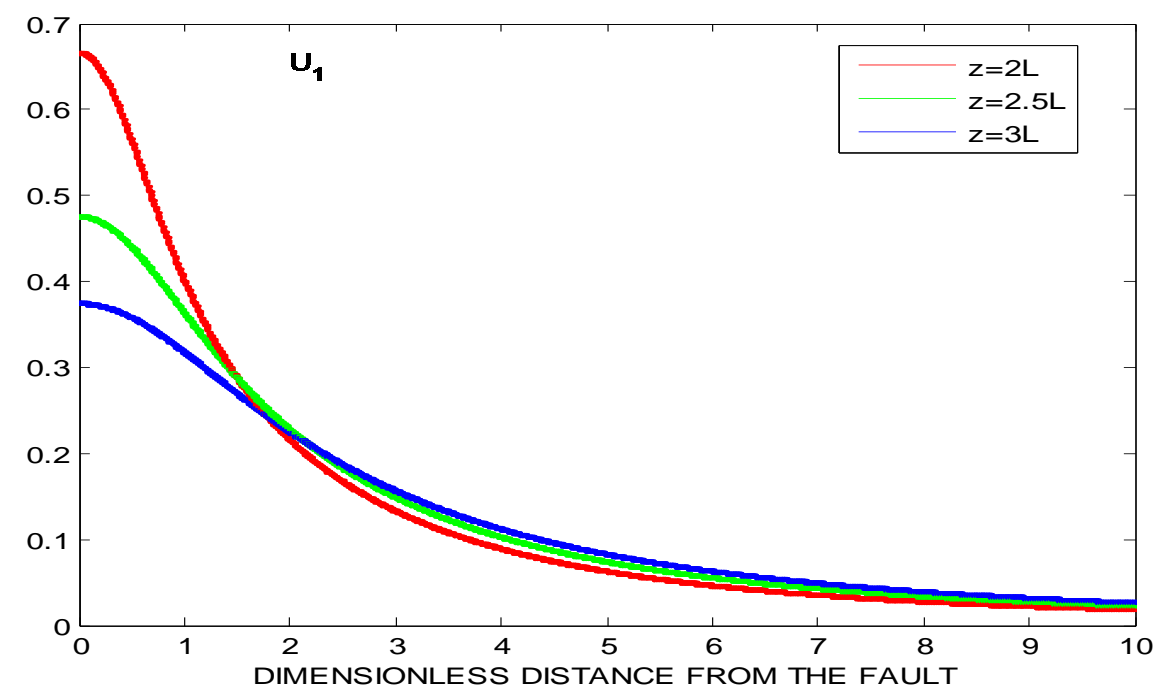

Fig. 4(a) Variation of dimensionless displacement $U_{1}$ with the distance from the fault

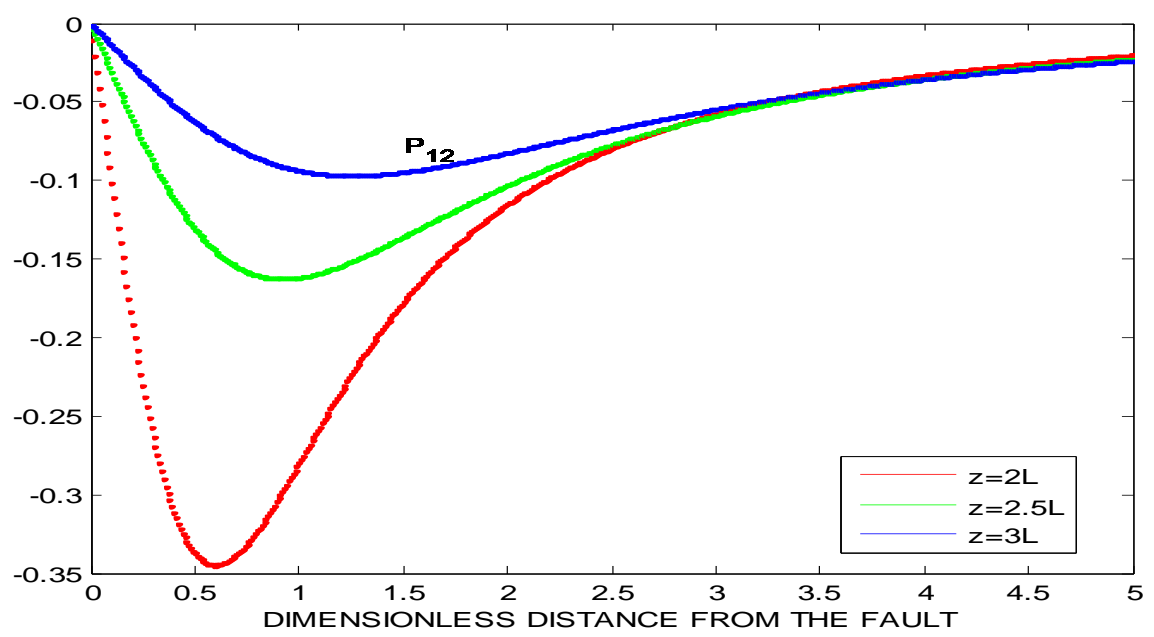

Fig. 4(b) Variation of dimensionless shear stress $\left(\mathrm{P}_{12}\right)$ with the distance from the fault 


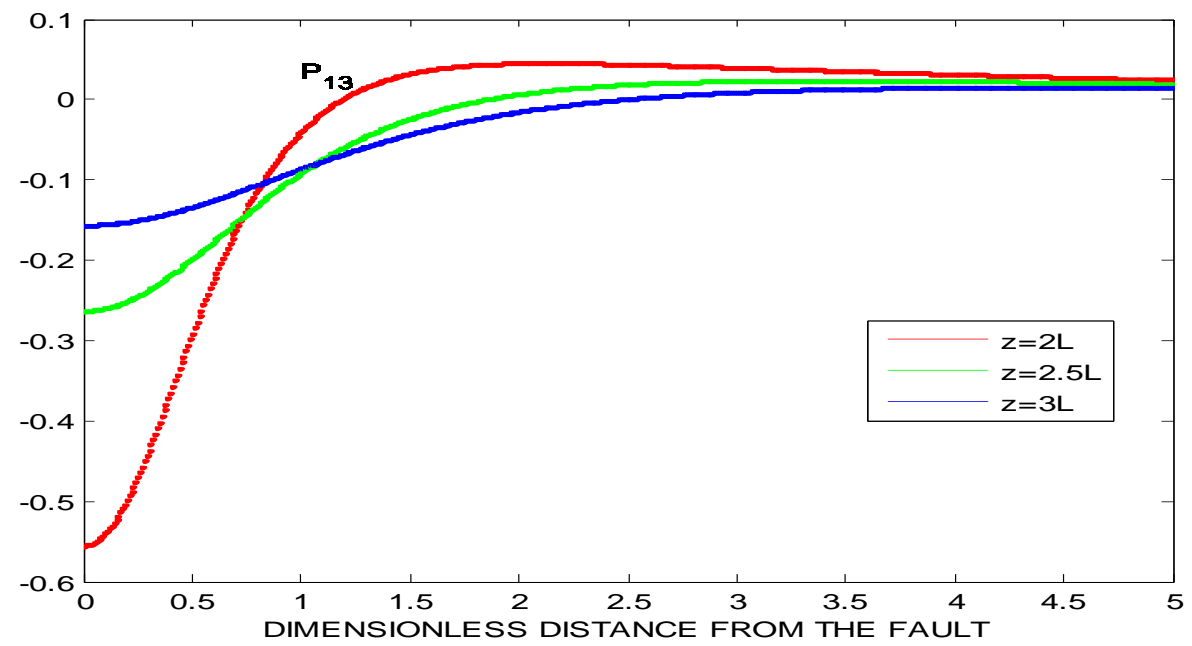

Fig. 4(c) Variation of dimensionless shear stress $\left(\mathrm{P}_{13}\right)$ with the distance from the fault

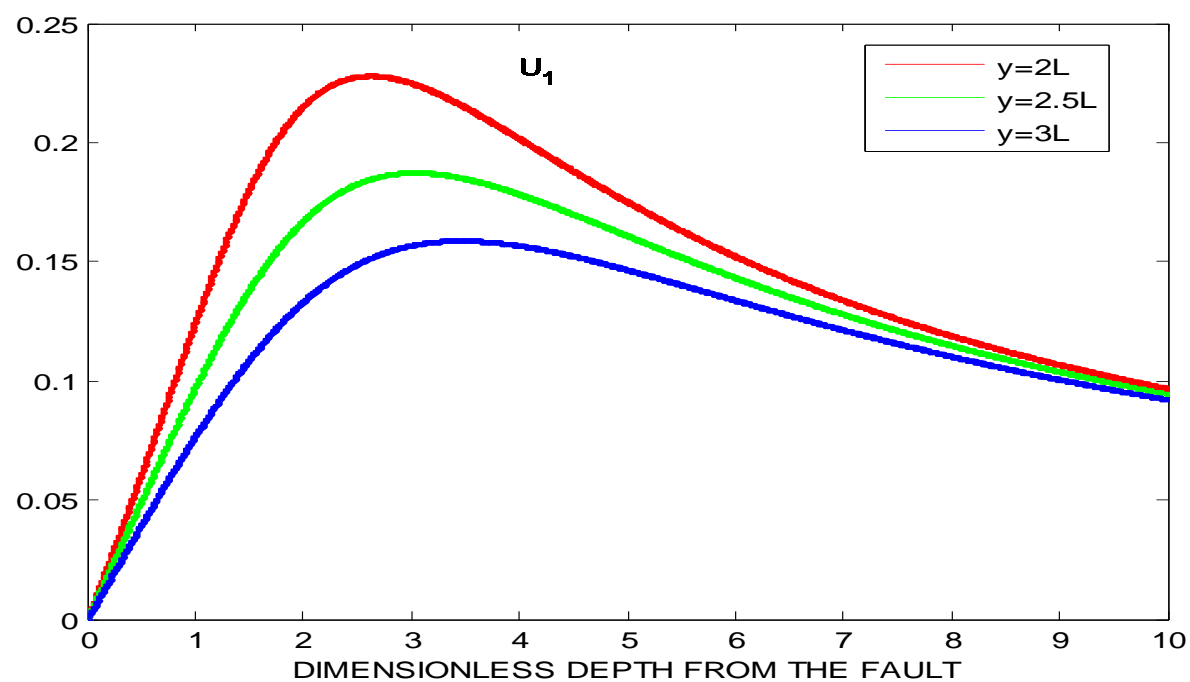

Fig. 5(a) Variation of dimensionless displacement $U_{1}$ with the depth from the fault

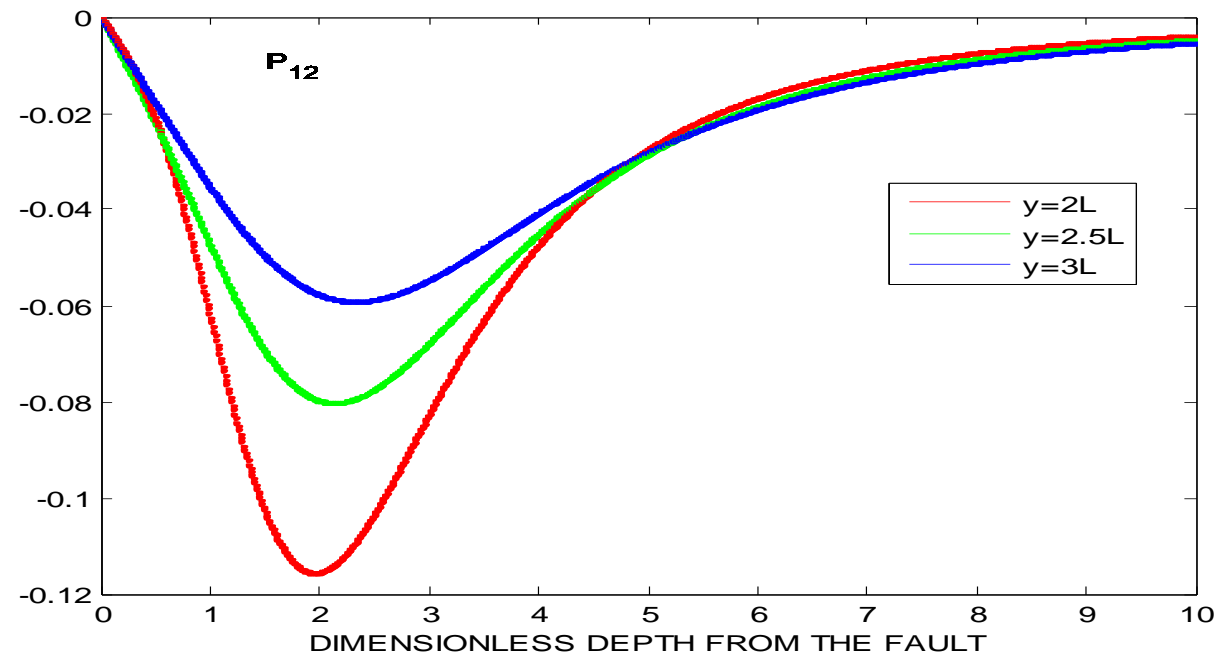

Fig. 5(b) Variation of dimensionless shear stress $\left(\mathrm{P}_{12}\right)$ with the depth from the fault 


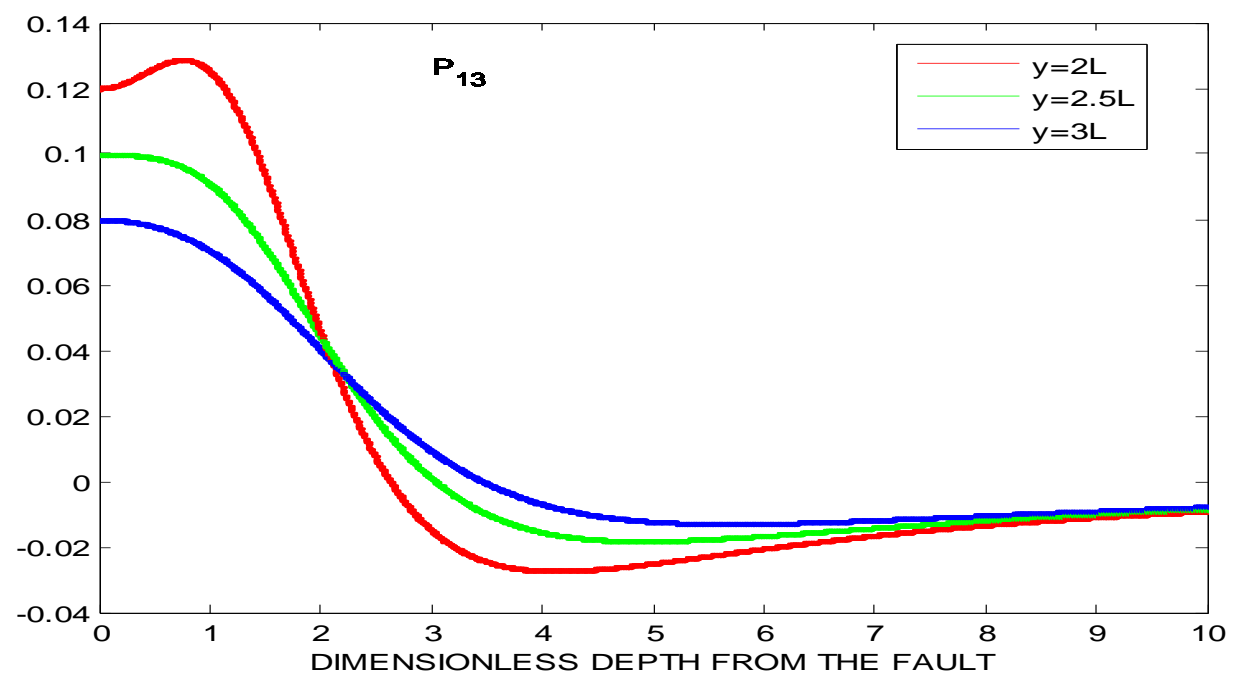

Fig. 5(c) Variation of dimensionless shear stress $\left(\mathrm{P}_{13}\right)$ with the depth from the fault

\section{References}

[1] Chinnery, M. A., The Deformation of the Ground Around Surface Faults, Bull. Seismol. Soc. Am., vol. 61, $1961,355-372$.

[2] Chugh, S., Madan, D. K. and Singh, K., Static Deformation Due To Non-Uniform Slip Along a Very Long Vertical Strike-Slip Geological Fault In Two- Phase Medium, Int. J. of Ecological Economics and Statistics, vol. 19, 2010, 47-65.

[3] Kumar, A., Singh, S. and Singh, J., Static Deformation of Two Welded Monoclinic Elastic Half-spaces due to a Long Inclined Strikeslip Fault, Proc.of Indian Acad. Of Sci-Earth and Planet. Sci., vol. 111, 2002, 125-131.

[4] Madan, D.K. and Garg, N.R., Static Deformation of an Orthotropic Horizontal Elastic Layer Coupling in Different ways to a base due to a very Long Inclined Strike-slip Fault Embedded in a Layer, Indian J. pure. Appl. Math., vol. 28, 1997, 697-712.

[5] Mahrer, K. D. and Nur, A., Strike-Slip Faulting in a Downward Varying Crust, J. Geophys. Res., vol. 84,1979a, $2296-2302$.

[6] Mahrer, K. D. and Nur, A., Static Strike-Slip Faulting in a Horizontally Varying Crust, Bull. Seismol. Soc. Am., vol. 69, 1979b, 9751009.

[7] Malik, M., Singh, M. and Singh, J., Static Deformation of a Uniform Half-space with a Rigid Boundary Due to a Vertical Dip- Slip Line Source, IOSR-J. Math., vol. 4, 2013, 26-37.

[8] Maruyama, T., On Two-dimensional Elastic Dislocations in an Infinite and Semi-infinite Medium, Bull. Earthq. Res. Inst., vol. 44, 1966, 811-871.

[9] McHugh, S. and Johnstone, M., Surface Shear Stress, Strain and Shear Displacement for Screw Dislocations in a Vertical Slab With Shear Modulus Contrast, Geophys. J. R. astr. Soc., vol. 49, 1977, 715-722.

[10] Mindlin, R.D. and Cheng, D. H., Nuclei of Strain in the Semi-Infinite Solid, J. Appl. Phys., vol. 21, 1950, 926-930.

[11] Press, F., Displacements, Strains and Tilts at Teleseismic Distances, J. Geophys. Res., vol. 70, 1965, $2395-2412$.

[12] Rani, S. and Singh, S., Static Deformation of Two Welded Half-spaces due to Dip-slip Faulting, Proc. Indian Acad. Sci.(Earth Planet. Sci.), vol. 101, 1992, 269-282.

[13] Rybicki, K., The Elastic Residual Field of a very Long Strike-Slip Fault in the Presence of Discontinuity, Bull. Seismol. Soc. Am., vol. 61, 1971, 79-92.

[14] Rybicki, K., Static Deformation of a Laterally Inhomogeneous Half-space by a Two-dimensional Strike-Slip Fault, J. Phys. Earth., vol. 26, 1978, 351-366.

[15] Rybicki, K. and kasahara, K., A Strike-Slip Fault in a Laterally Inhomogeneous Medium, Tectonophysics, vol. 42, 1977, 127-138.

[16] Sato, R., Crustal Deformation due to Dislocation in a Multilayered Medium, J. Phys. Earth, vol. 19,1971, 31 - 46.

[17] Sato, R. and Yamashita, T., Static Deformation in an Obliquely Layered Medium, Part II: Dip-slip Fault, J. Phys. Earth., vol. 23, $1975,113-125$.

[18] Sharma, R. K. and Garg, N. R., Subsurface Deformation of a Layered Half-space Due to a very Long Strike-Slip Dislocations, Proc. Ind. National Sci. Acad.

[19] Singh, J., Malik, M. and Singh, M., Deformation of a uniform half-space with rigid boundary due to long tensile fault, ISET Journal of Earthquake Technology., vol. 48, 2011.

[20] Singh, S. J., Static Deformation of a Multilayered Half-space by Two-dimensional sources, Acta Geophys. Pol., vol. 33, 1985, 123134.

[21] Singh, S. J. and Garg, N.R., On Two-dimensional Elastic Dislocations in a Multilayered Half-space, Phy. Earth Planet. Int., vol. 40, $1985,135-145$.

[22] Singh, S.J. and Garg, N.R, On the Representation of Two-Dimensional Seismic Sources, Acta Geophys. Pol., vol. 34, $1986,1-12$.

[23] Singh, S.and Rani, S., Static Deformation due to Two-dimensional Seismic Sources Embedded in an Isotropic Half-space in Welded Contact with an Orthotropic Half space, J. Phys. Earth, vol. 39,1991, 599-618.

[24] Singh, S. J., Rani, S. and Garg, N. R., "Displacements and Stresses in Two Welded Half-spaces Caused by Two Dimensional Sources, Phys. Earth Planet. Int., vol. 70, 1992, 90-101.

[25] Sokolnikoff, I. S., Mathematical Theory of Elasticity, (Newyork: McGraw-Hill), 1956.

[26] Steketee, J.A, On Volterra's Dislocations in a Semi-infinite Elastic Medium, Can. J. Phys., vol. 36,1958a, 192-205.

[27] Stekettee, J.A, Some Geophysical Applications of the Elasticity Theory of Dislocations, Can. J. Phys., vol. 36, 1958b, 1168-1198.

[28] Wang, R. and Wu, H. L., Displacement and Stress Fields Due to a Non-uniform Slip Along a Strike-Fault, Pure Appl. Geophys., vol. $121,1983,601-609$ 


\section{Appendix I}

$\left[z>0, y^{2}+z^{2}=R^{2}\right]$
i. $\quad \int_{0}^{\infty} e^{-k z} \frac{\sin k y}{k} d k=\tan ^{-1}\left(\frac{y}{z}\right)$
vi $\int_{0}^{\infty} e^{-k z} \cos k y k d k=\frac{1}{R^{2}}\left(\frac{2 z^{2}}{R^{2}}-1\right)$
ii. $\quad \int_{0}^{\infty} e^{-k z} \frac{\cos k y}{k} d k=-\log R$
vii $\int_{0}^{\infty} e^{-k z} \sin k y k^{2} d k=\frac{2 y}{R^{4}}\left(\frac{4 z^{2}}{R^{2}}-1\right)$
iii. $\quad \int_{0}^{\infty} e^{-k z} \sin k y d k=\left(\frac{y}{R^{2}}\right)$
viii $\int_{0}^{\infty} e^{-k z} \cos k y k^{2} d k=\frac{2 z}{R^{4}}\left(\frac{4 z^{2}}{R^{2}}-3\right)$
iv. $\quad \int_{0}^{\infty} e^{-k z} \cos k y d k=\left(\frac{y}{R^{2}}\right)$
ix $\int_{0}^{\infty} e^{-k z} \sin k y k^{3} d k=\frac{24 y z}{R^{6}}\left(\frac{2 z^{2}}{R^{2}}-1\right)$
v. $\quad \int_{0}^{\infty} e^{-k z} \sin k y k d k=\frac{2 y z}{R^{4}}$
x $\quad \int_{0}^{\infty} e^{-k z} \cos k y k^{3} d k=\frac{6}{R^{4}}\left(\frac{8 z^{4}}{R^{4}}-\frac{8 z^{2}}{R^{2}}+1\right)$ 